\title{
Utilizing Optical Monitoring to Predict the Effluent Quality in the Activated Sludge Processes
}

\author{
${ }^{1}$ Control Engineering, University Oulu; *jani.tomperi@oulu.fi
}

Jani Tomperi*

\begin{abstract}
The optical monitoring device was used for imaging industrial and municipal activated sludge processes. The results were utilized to predict the important effluent quality parameters (BOD, COD, N, P, SS) indicating the efficiency of the wastewater treatment processes. The optimal subsets of variables for each model were searched using mathematical variable selection methods. The models based on optical monitoring and process variables from the early stage of the treatment process can be used to predict the effluent quality hours in advance compared with traditional measurements, which enables the optimization of process control, keeping the process in a stable operating condition and avoiding environmental risks and excessive operation costs.
\end{abstract}

\section{Introduction}

A wastewater treatment process should efficiently remove oxygen-demanding substances, excessive nutrients and toxicants from wastewater. Both industrial and municipal wastewaters are most commonly treated in complex biological activated sludge processes (ASPs) where the operation of the treatment process is affected by several physical, chemical and microbiological factors. In an ASP, flocculation is in a critical role and the key element for the efficient operation of an ASP is a good bacterial balance in biomass, which is very sensitive to internal and external disturbances such as major changes in the quality and quantity of the influent.
The disturbances in the bacterial balance are most often shown as dysfunctional flocculation and settling properties. Recovering from the disturbances is slow which causes long-lasting challenges to process control and possible environmental damages as low-quality effluent is discharged to waterways [1-3].

More attention must be focused on the optimal operation of the wastewater treatment processes to meet the limitations to effluent discharges and keeping operating costs decent. Predictive information on the status of the process and the effluent quality enable the optimization of the process operation and thereby avoiding environmental risks and excessive operation costs. Conventional process measurements and expert knowledge, that are important in process operation, are, however, not adequate to give the early warnings of changes in the treated wastewater quality. Therefore, new measurement devices and methods are required.

Automatic optical monitoring of floc morphology characteristics in the ASP yields fast new objective information about the quality of the treatment process and reveals the reasons for settling problems. In addition, combined to predictive modelling, the quality of the treated wastewater is shown in advance, hours before problems occur and would be noticed by traditional process measurements [4-7]. This valuable predictive information can be used as assistance in optimizing the process control.

Study of utilizing the automatic optical monitoring of activated sludge process combined with mathematical variable selection and modelling have been carried out in the industrial and the municipal wastewater treatment processes, and are summarized in this paper. 


\section{Material and Methods}

\subsection{Industrial wastewater treatment plant}

The industrial wastewater treatment process treats in addition to the pulp making process wastewaters from two chemical processes located in the pulp and paper mill area. The average wastewater flow through the treatment process is around $30000 \mathrm{~m}^{3} /$ day. The collected data consisted of optical monitoring results and selected process measurements including the effluent quality parameters from a period of 13 consecutive months. The optical monitoring was carried out by regular but sparse intervals and therefore the dataset included only 54 measurement times.

\subsection{Municipal wastewater treatment plant}

The municipal WWTP treats mainly wastewaters from domestic sources $(85 \%$ of the influent flow). WWTP uses mechanical, biological and chemical processes to treat the wastewater of over 800,000 inhabitants. The average daily flow is $280,000 \mathrm{~m}^{3}$ of wastewater. The optical monitoring device was sited at one of the nine parallel activated sludge process lines. The collected data consisted of daily optical monitoring results, online process measurements, and various quality parameters that were measured by collecting grab samples only two to three times a week during a period of 16 months. Thus, the total amount of data was 94 measurement times.

\subsection{Optical monitoring and image analysis}

To replace a laborious, slow and subjective method to study wastewater samples manually using a microscope, a small-scale automatic optical monitoring device was designed especially for in-situ use [8]. The optical monitoring device was developed and tested in a laboratory and at a municipal wastewater treatment plant over an one-year period, and proved functional for reliable in-situ monitoring of floc morphology $[4,6]$.

The optical monitoring device (Figure 1) consists of the imaging unit, the sample handling unit and the control PC and electronics unit. The control PC and electronics unit controls the pump and valves synchronized with image acquisition. The wastewater samples taken from the aeration tank of ASP are diluded and pumped through the cuvette of the imaging unit. A cuvette is illuminated and imaged with a high resolution chargecoupled device (CCD) camera.

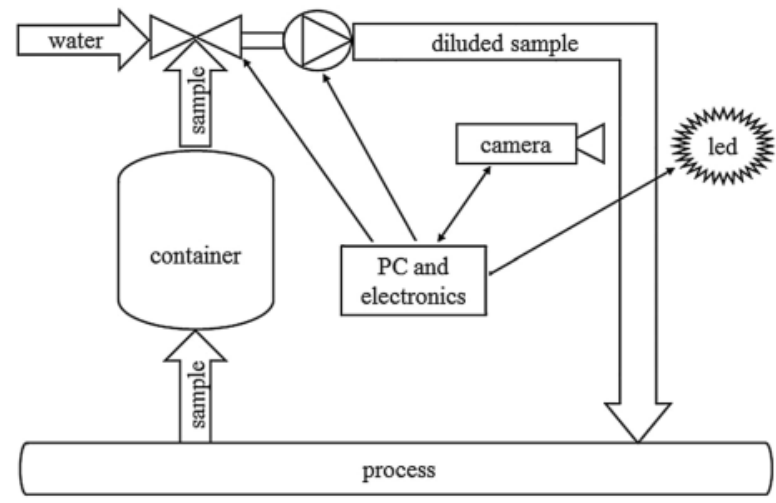

Figure 1: The online optical monitoring device for imaging activated sludge process [6].

The automated image analysis program analyzes the morphological parameters of flocs and filaments from the wastewater sample videos that consist of hundreds of images. The image analysis programme calculates and analyses various size (equivalent diameter, floc area, filament length, etc.) and shape (fractal dimension, form factor, roundness, etc.) parameters of each particle in each image. The parameters are calculated as an average of the values for individual objects over a single image. Image processing and analyses methods and the mathematical formulas of the calculated size and shape parameters are presented in detail in [8].

\subsection{Variable selection and modelling}

The measured optical monitoring variables were used together with the process measurements to develop predictive models for the traditional quality variables of treated wastewater including suspended solids (SS), eutrophication in waterways causing nitrogen $(\mathrm{N})$ and phosphorus (P), chemical oxygen demand (COD) and biochemical oxygen demand (BOD) that indicate the amount of dissolved oxygen required to oxidize the organic substances in wastewater [1]. All the data processing, variable selection and modelling were performed using Matlab (The MathWorks Inc.) and selfdeveloped scripts.

At first, dataset was inspected and unrealistic values were deleted and replaced with linear interpolation. Before variable selection and modelling, the dataset was scaled between $[-2,+2]$ using the nonlinear scaling method based on generalized moments, norms and skewness (presented in more detail in [9]). 
Five mathematical variable selection methods (correlation, stepwise, forward, genetic algorithm (GA), successive projections algorithm (SPA) + GA) that did not take into account any deterministic models or chemical or biological knowledge about the activated sludge process, were used to find the optimal subsets of input variables for the models. Variable selection is important step to reduce the number and choose the optimal input variables that have significant relationships with the output variable but are not strongly correlated to each other and do not include noise. Using too many input variables increase the risk to develop an over-fitted model which has an excellent training result but is not usable with a new upcoming data. For a very large dataset one variable selection method can be used for the variable elimination before the final variable selection by another method. The more detailed backgrounds of the above mentioned variable selection methods are presented for example in [6].

The quality of the developed model depends highly on the quality and length of the dataset. Data should include a sufficient number of samples and it should be fully representative of the full spectrum of all possible conditions. For efficient training and validation, both subsets of the data have to be long and representative enough of all possible conditions. Splitting the dataset to two subsets may cause a significant loss of data in training the model. Due to the small size of the dataset available in this study, a static split into the training and validation subsets of data was not a feasible approach. Instead, a 5-fold cross-validation was used to predict the fit of a model for a validation set without an explicit validation set. In k-fold cross-validation, the whole data is used for training and validating the model by randomly partitioning the dataset into $\mathrm{k}$ subsets of equal size and using k-1 parts of the data for training and one part for validation and repeating this $\mathrm{k}$ times until each of the subsets is used once as the validation data. Final estimation is produced by combining these $\mathrm{k}$ results of the folds $[10,11]$. In this study, multivariable linear regres- sion (MLR) was utilized to predict the output variable as a linear combination of selected input variables and the performance of the model was evaluated by the coefficient of determination $\left(\mathrm{R}^{2}\right)$ and Root Mean Square Error (RMSE).

\section{Results and Discussion}

The results of utilizing the automatic optical monitoring of activated sludge process combined with mathematical variable selection and modelling in the industrial [7] and municipal $[6,12]$ wastewater treatment plants are summarized in the following. It is important to bear in mind that the results based solely on a mathematical analysis may not accurately correspond the actual situation in the wastewater treatment process. The complexity of a wastewater treatment process easily causes quasicorrelations. A high correlation between variables not always mean strong real-world causality and there may be also many hidden factors that affect in the real process but are not shown in the data analysis due to the limited amount of data or measurements and due to the analysis method.

The best modelling results (industrial and municipal WWTP) are presented in Table 1 . In both studies, input variables selected by the GA method resulted the best models. However, for example forward selection, that is computationally much faster method that GA, yielded almost as good results. The behaviour of effluent COD model in the industrial WWTP is presented in Figure 2 and the behaviour of the effluent SS model in the municipal WWTP is presented in Figure 3. The results show that the models are not perfect but generally good and accurate to show the level and changes of the effluent quality variables in various operation conditions. The earlier studies have stated that based on the optical monitoring the settling problem in the industrial WWTP occurred during the study period was most likely caused by dispersed growth [13] and in the municipal ASP the poor settling was caused by filamentous bulking [4].

\begin{tabular}{lllllllllll}
\hline & BOD & \multicolumn{3}{c}{ COD } & & $\mathrm{N}$ & & $\mathrm{P}$ & & SS \\
& $\mathrm{R}^{2}$ & $\mathrm{RMSE}$ & $\mathrm{R}^{2}$ & $\mathrm{RMSE}$ & $\mathrm{R}^{2}$ & $\mathrm{RMSE}$ & $\mathrm{R}^{2}$ & $\mathrm{RMSE}$ & $\mathrm{R}^{2}$ & RMSE \\
\hline $\begin{array}{l}\text { Industrial WWTP } \\
\text { (GA subset) }\end{array}$ & 0.71 & 0.69 & 0.76 & 0.50 & 0.69 & 0.63 & 0.58 & 0.74 & 0.67 & 0.58 \\
$\begin{array}{l}\text { Municipal WWTP } \\
\text { (GA subset) }\end{array}$ & 0.55 & 0.64 & 0.55 & 0.64 & 0.63 & 0.61 & 0.69 & 0.52 & 0.79 & 0.47 \\
\hline
\end{tabular}

Table 1: The best modelling results for the industrial and municipal WWTP effluent quality parameters using input variables selected by GA. Modified from $[6,7]$. 
It was also discovered that according to the data analyses the municipal process is more temperature related than the industrial. The effluent quality has a clear seasonal pattern correlating the wastewater temperature $[6,7]$.

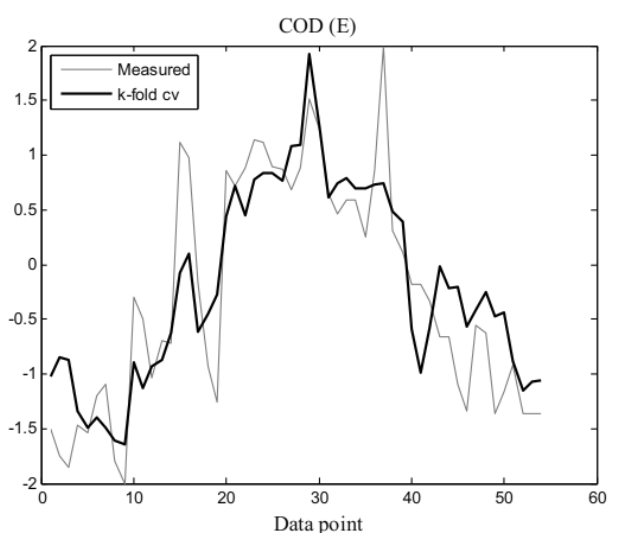

Figure 2: Model for effluent COD at the industrial WWTP [7].

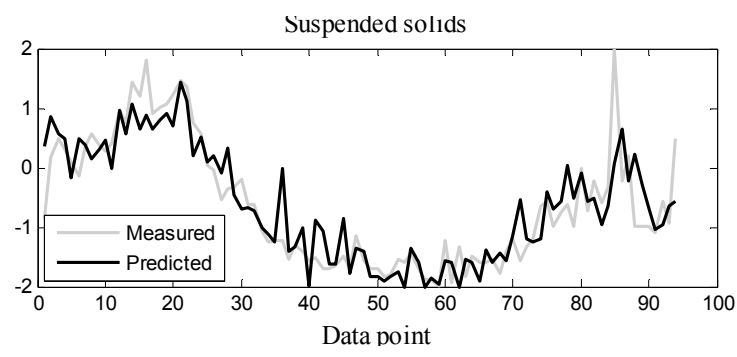

Figure 3: Model for effluent SS at the municipal WWTP [6].

\section{Conclusions}

The optical monitoring device is a valuable tool for monitoring the changes in floc morphology. The objective, continuous and fast method includes several morphological characterization variables and enables observing the changes in the wastewater quality. Combined to predictive modelling it has potential to be utilized in controlling the process, keeping the process in stable operating conditions and avoiding environmental risks.

\section{References}

[1] Tchobanoglous G, Burton FL, Stensel HD. Wastewater engineering: treatment and reuse. 4th ed. Boston: McGraw-Hill Education; 2003. 1819 p.

[2] Amaral AL, Ferreira EC. Activated sludge monitoring of a wastewater treatment plant using image analysis and partial least squares regression. Anal. Chim. Acta. 2005;544:246-253.
[3] Mesquita DP, Dias O, Amaral AL, Ferreira EC. Monitoring of activated sludge settling ability through image analysis: validation on full-scale wastewater treatment plants. Bioprocess Biosyst Eng. 2009;32:361-367.

[4] Koivuranta E, Stoor T, Hattuniemi J, Niinimäki J. Online optical monitoring of activated sludge floc morphology. J Water Process Eng. 2015;5:28-34. doi:10.1016/j.jwpe.2014.12.009

[5] Tomperi J, Koivuranta E, Kuokkanen A, Juuso E, Leiviskä K. Real-time optical monitoring of the wastewater treatment process. Environ Technol. 2016;37(3):344-351. doi:10.1080/09593330.2015.1069898

[6] Tomperi J, Koivuranta E, Kuokkanen A, Leiviskä K. Modelling effluent quality based on a real-time optical monitoring of the wastewater treatment process. Environ Technol. 2017;38(1):1-13. doi:10.1080/09593330.2016.1181674

[7] Tomperi J, Koivuranta E, Leiviskä K. Predicting the effluent quality of an industrial wastewater treatment plant by way of optical monitoring. Journal of Water Process Engineering. 12017;6:283-289. doi:10.1016/j.jwpe.2017.02.004

[8] Koivuranta E, Keskitalo, Haapala A, Stoor T, Sarén M, Niinimäki J. Optical monitoring of activated sludge flocs in bulking and non-bulking conditions. Environ Technol. 2013;34(5-8):679-686. doi:10.1080/09593330.2012.710410

[9] Juuso E. Integration of intelligent systems in development of smart adaptive systems: linguistic equation approach. Acta Universitatis Ouluensis. Series C, Technica 476. Oulu. Dissertation. 258. 2013. http://urn.fi/urn:isbn:9789526202891

[10] Rao RB, Fung G, Rosales R. On the dangers of crossvalidation: an experimental evaluation. In SIAM Data Mining, Philadelphia, PA. 2008.

[11] Arlot S, Celisse A. A survey of cross-validation procedures for model selection. Stat Surv. 2010;4:40-79.

[12] Tomperi J, Leiviskä K. Comparison of modelling accuracy with and without exploiting automated optical monitoring information in predicting the treated wastewater quality. Environ. Technol. 2018;39(11):1442-1449. doi:10.1080/09593330.2017.1331267.

[13] Koivuranta E, Keskitalo J, Stoor T, Hattuniemi J, Sarén M, Niinimäki J. A comparison between floc morphology and the effluent clarity at a full-scale activated sludge plant using optical monitoring. Environ. Technol. 2014;35(13): 1605-1610. doi:10.1080/09593330.2013.875065.

Note. The data were collected during the Measurement, Monitoring and Environmental Efficiency Assessment (MMEA) research programme. Aki Sorsa (D.Sc. (Tech.)) is acknowledged for help in variable selection issues and Elisa Koivuranta (D.Sc. (Tech.)) is acknowledged for producing the original optical monitoring data. 